\title{
RHIC BEAM COMPONENTS IMPEDANCE ANALYSIS WITH MAFIA
}

\author{
H. Wang ${ }^{*}$, W. Guryn, H. Huang, D. Trbojevic, BNL, Upton, NY 11973, USA
}

\section{Abstract}

The RHIC CNI polarimeter chambers and Roman Pots of PP2PP's detector have been simulated by a 3D electromagnetic field code MAFIA [1] to calculate the narrowband shunt impedance and the beam parasitic loss in frequency domain. The field profiles in high order modes (HOMs) of the polarimeter were then confirmed by bead-pull measurement. With an optimum design, the shunt impedances for both components are under the limits set by the coupled bunch instability consideration.

\section{IMPEDANCE LIMIT}

The high intensity $\left(2 \times 10^{11}\right.$ proton/bunch $)$ and short bunch ( $0.24 \mathrm{~ns}$ at storage) beam in RHIC can generate wakefield inside the beam components. The long-range wakefield is more harmful for RHIC longitudinal beam dynamics. In other words, a beam component with high shunt impedances in narrowband HOMs could cause beam emittance blowup, unstable or even lost. Consequently, the beam components require a careful impedance analysis to fit into RHIC impedance budget.

There have been two independent calculations to set the impedance limit for the proton beam circulation based on the thresholds of coupled bunch instability. One was using the ZAP tracking code [2], for both injection and storage cavities with dipole modes only. The bunch area limit was set $0.3 \mathrm{eV}$-sec [3] with a growth rate of $2 \mathrm{sec}^{-1}$. Another was done analytically for injection cavity but included both dipole and quadruple modes [4]. The limit was based on the 2.8 ns RMS bunch length and $2 \mathrm{sec}^{-1}$ growth rate. We have modified this analytic output to include extra sextuple and octupole modes for a more cautious limit [5].

The simplest guideline to adopt for this analysis is to let the collective impedances of beam components not exceed these limits set for the accelerator cavities.

\section{BEAM COMPONENTS}

Two beam components have been done in this analysis, the target chamber (Figure 1) of CNI polarimeter [6] and the Roman Pots (Figure 2) of the PP2PP's detector.

The polarimeter chamber is $1.6 \mathrm{~m}$ long and $16 \mathrm{~cm}$ in radius. Six silicon strip detectors installed at $90^{\circ}$ and $45^{\circ}$ from vertical axis and apart and their ports sunk into the chamber. Two target assemblies orientated in $\mathrm{X}$ and $\mathrm{Y}$. Each one holds 3 wire targets, $3 \mathrm{~cm}$ long each, by the beam path. The chamber radius is compromised between the room required by the target feeding operation and impedance reduction. An analytical calculation on a pillbox for monopole modes has shown the scale of the

*Current e-mail: haipeng@jlab.org shunt impedance with radius [7]. A $10 \%$ short in radius may lead to a $15 \%$ impedance reduction. The target box length is $50.8 \mathrm{~cm}$. A 5:1 conical tapering structure is attached at each end of target box for a further impedance reduction. The ratio is the length to the difference of the pipe radii. The " $5: 1$ " tapering rule applies to all RHIC beam components aperture transitions [8].

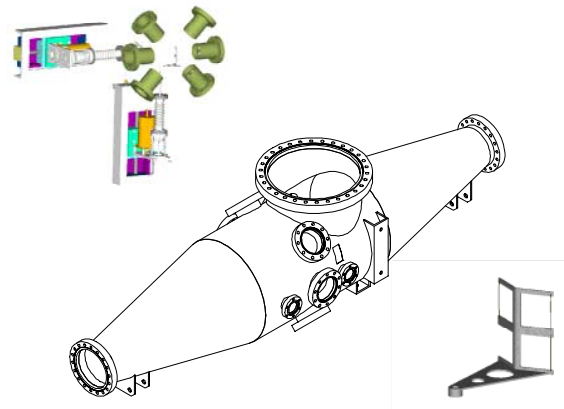

Figure 1: CNI polarimeter chamber (center), target holder (low right), detector ports and target assemblies (up left). Particle beam goes from the left to the right.

The Roman Pots geometry is rather simple. Two rectangular pots $\left(5^{\prime \prime} \times 1.6^{\prime \prime}\right)$ will insert into the cylinder chamber with a minimum gap of $2 \mathrm{~cm}$. The pot can be pulled up, so a cavity will form inside. The chamber length is $99 \mathrm{~cm}$ and radius is $9.9 \mathrm{~cm}$.

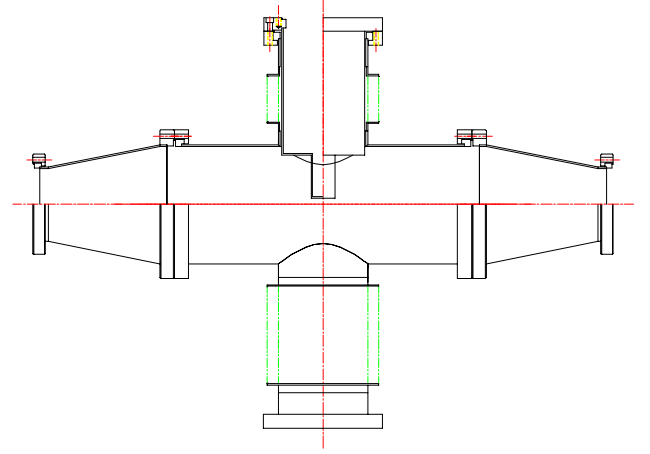

Figure 2: The Roman Pots of PP2PP's detector.

\section{MAFIA ANALYSIS}

The E module of MAFIA has been used for many RHIC beam component impedance calculations. We followed a similar method but a careful strategy. As the first step, a simple pillbox cavity was analyzed, whose impedance can be solved analytically. Two MathCAD programs for TM and TE modes were first used to get the numerical calculations. The integration results for TM monopole modes agree with formulae in [7]. Then up to 60 modes were calculated by MAFIA. 50 modes solutions with the highest frequency $1.612 \mathrm{GHz}$ were stored. The E module can check solution accuracy of each mode with Maxwell's 
equations after final iteration. Four bad solutions were found. All of them are not physical by comparing with analytical results. Five non-zero shunt impedance modes are TM010 TM014 monopole modes. Comparing with MathCAD, overall impedance value discrepancy is within $10 \%$. All other 41 modes found are TE modes or degenerated TM modes, with zero shunt impedances. Among them, three were monopole TE, 20 were dipole and higher azimuthal TE and TM modes. The azimuthal modes gave redundant solutions except two modes near the $1.6 \mathrm{GHz}$. The redundant solutions have very little difference in parameters. A detail examination reveals their field patterns being just about $\mathrm{X}$ or $\mathrm{Y}$ symmetric. That is due to no symmetry plane putting in the simulation. Comparing all good solutions with MathCAD calculation, the agreement for quality factors are within $7 \%$, and for frequencies are within $1 \%$. In summary, we found that MAFIA 3D simulation gave us reliable results except the bad solutions.

In the second step, the target box with all interior components was simulated to compare with same size pillbox. The result shows all monopole TM modes have high peaks, but rather reduced. The impedance peaks split into two from TM011 mode and up. All TM modes shift higher frequencies except the TM011. The TE modes show up in low peaks. All these changes can be explained by the perturbation of interior components to the pillbox.

In the third step, the 5:1 tapers were added to the target box. The tapers bring down the narrowband peaks mainly at high frequency band. Figure 3 shows the comparison and the impedance limits developed early. During this step, we found the target frames have to be insulated from the cavity wall. Otherwise, even with the tapers, the impedance at $200 \mathrm{MHz}$ will exceed the limit. The target frame with a metal "V" plate holder acts like a conducting membrane plane, which enhances the monopole electric fields. We had to change the design to a ceramic plate. Figure 4 shows their difference. The simulations for target in three positions were also carried out. Figure 5 shows these variations to assure the impedances are under limits.

In the final step, we put the large view port and a screen in the simulation. The screen has a 6-inch hole of mesh area. The mesh hole size in the simulation is about $0.5 \mathrm{~cm}$ $\times 0.5 \mathrm{~cm}$. The purpose of the screen is to reduce the broadband impedance induced by the view port [9]. The simulation was for narrowband. So the result indicated no difference from the closed cylinder or a solid screen. A non-physical mode, which was leaking out of screen, was found also a bad solution. Over all in general, one typical simulation run for a whole polarimeter used about 2 $\mathrm{mesh} / \mathrm{cm}$ in $\mathrm{X}-\mathrm{Y}$ cross section in average. In $\mathrm{Z}$ dimension, about $2 \mathrm{mesh} / \mathrm{cm}$ was used in target box section and 1 $\mathrm{mesh} / \mathrm{cm}$ in tapering sections. The total mesh nodes were about 1 million. The CPU time on the BNL SUN2 workstation was about $17 \mathrm{hrs}$ for a 40 -modes calculation.

RHIC yellow ring polarimeter has two more ports than blue ring's. A simulation shows the impedance has no apparent difference from the blue one.

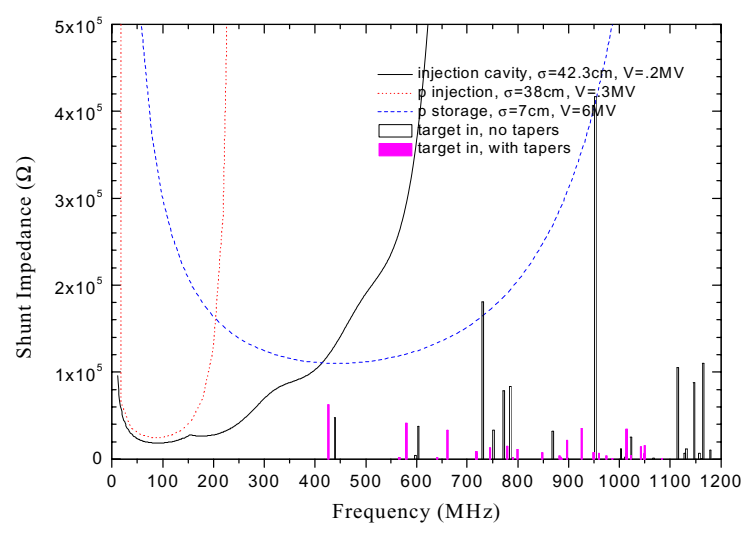

Figure 3: Comparison between with and without tapers.

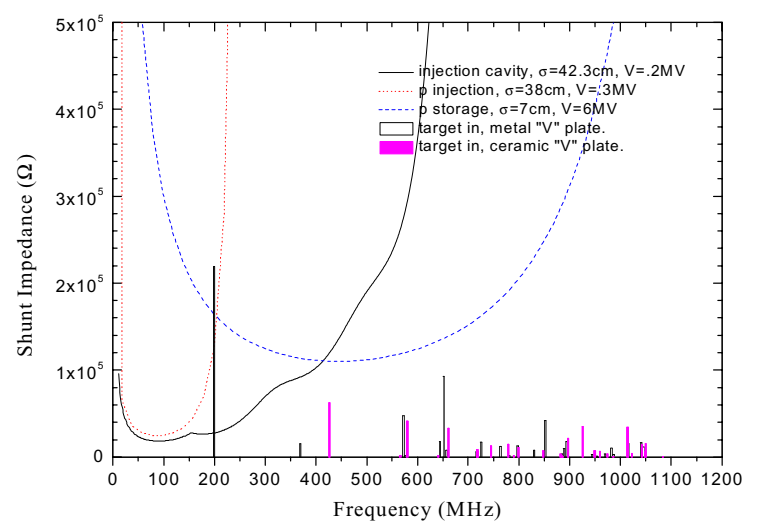

Figure 4: Comparison between ceramic and metal "V" plates.

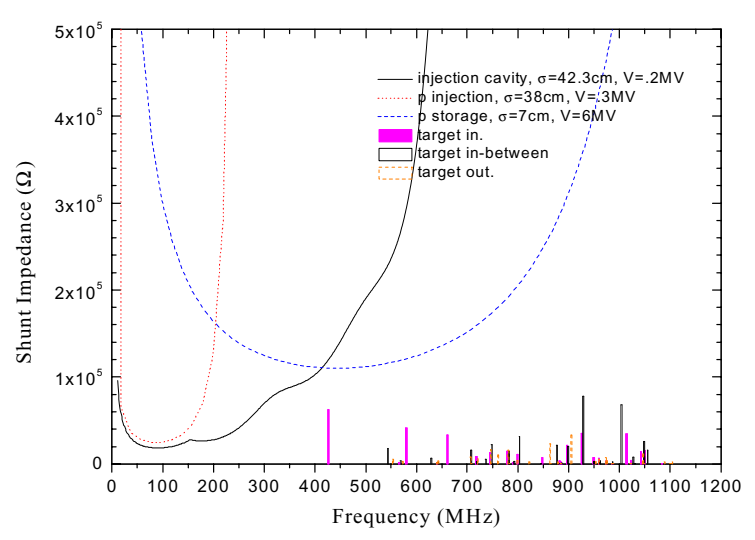

Figure 5: Comparison with different target positions.

The simulation for Roman Pots was simpler than the polarimeter. Four runs to simulate the pot in different positions show the impedance is much less than the polarimeter (see Figure 6).

\section{BEAM PARASITIC LOSS}

A bunched beam can lose certain energy when traversing a component due to its shunt impedance. The 
average power loss per beam cycle for Gaussian bunches with a RMS length $\sigma_{z}$ is

$$
P_{\text {ave }}\left(\sigma_{z}\right)=\frac{N_{b} c q^{2}}{4 R_{0}} \sum_{i=0}^{28} f_{i} \frac{R_{s i}}{Q_{s i}} \exp \left[-\left(\frac{2 \pi f_{i} \sigma_{z}}{c}\right)^{2}\right] \text { (1) }
$$

Here we ignore the wakefield between bunches and assume the energy filling time to the cavity is about same as the damping time. The $q$ is total charge in bunch, $c$ is the speed of light, $R_{s i}$ is shunt impedance, $Q_{i}$ is quality factor, $f_{i}$ is resonance frequency. Note $i$ is for $i$ th mode. $R_{0}$ is average radius of collider ring, $N_{b}$ is bunch number in the ring. The sum covers 29 modes calculated by MAFIA. The metallic loss is $R / Q$ or geometry dependent only. Figure 7 gives the calculation from (1) for the polarimeter. We can see that the metal "V" plate was neither acceptable for the instability nor for the heat loss here. The 5:1 tapers only relax the power loss when $\sigma_{z}<0.12 \mathrm{~m}$. The ceramic "V" plate was going to use MACRO ${ }^{\mathrm{TM}}(\tan \delta$ $=0.01$ ) because it can be machined. The dielectric loss relative to the total was still large. For example, it was 61 $\%$ for TE111 mode and 35\% for TM010 mode. That gave $\sim 73 \mathrm{~W}$ heat on each plate. After this calculation, the $\mathrm{Al}_{2} \mathrm{O}_{3}$ ( $\tan \delta \approx 0.0003$ ) was used, so the dielectric loss can be reduced to $\sim 2 \mathrm{~W}$. Total metal loss power was $\sim 94 \mathrm{~W}$, which dissipates over large chamber surface. The deposit density is only $\sim 8 \mathrm{~mW} / \mathrm{cm}^{2}$. No cooling is needed.

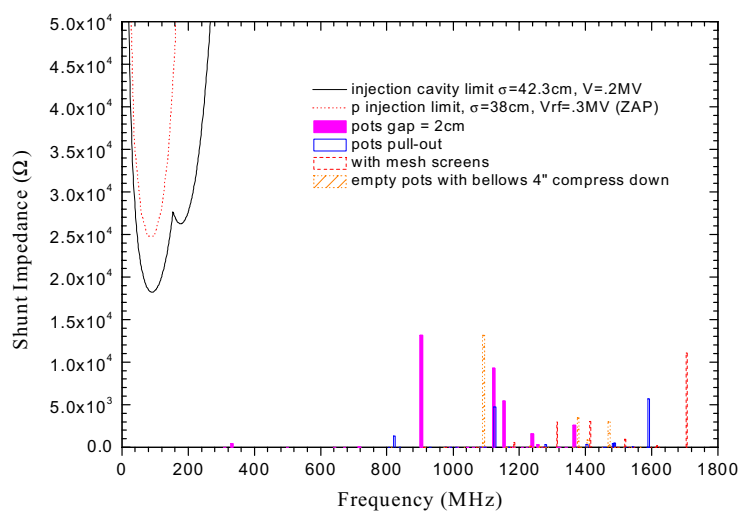

Figure 6: Roman Pot of PP2PP detector impedance with different operation positions.

\section{BEAD-PULL MEASUREMENT}

To confirm the MAFIA analysis, an on-axial bead-pull measurement was done before the polarimeter was online. A ceramic bead was used. A simple phase-lock circuit was set to measure the resonance frequency change due to the bead perturbation. We have selected numbers modes to measure. The comparison with MAFIA for TM010 mode is shown in Figures 8. The left coordinate is normalized MAFIA field. The right coordinate is the bead-pull data calculated from the frequency change. Due to large size of the bead, both longitudinal and transverse electric fields can contribute to measured data. The "bump" on the shoulder of the curve shown in the Figure
8 was assured by MAFIA is due to the transverse field contribution.

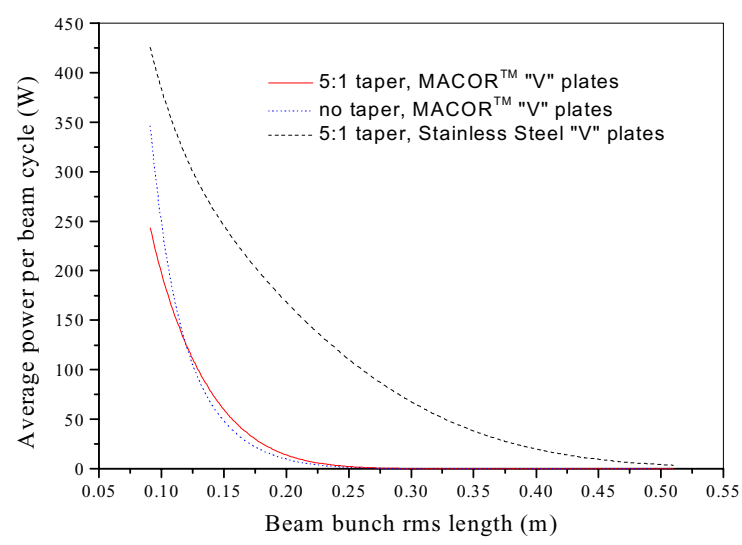

Figure 7: Average beam power loss to polarimeter.

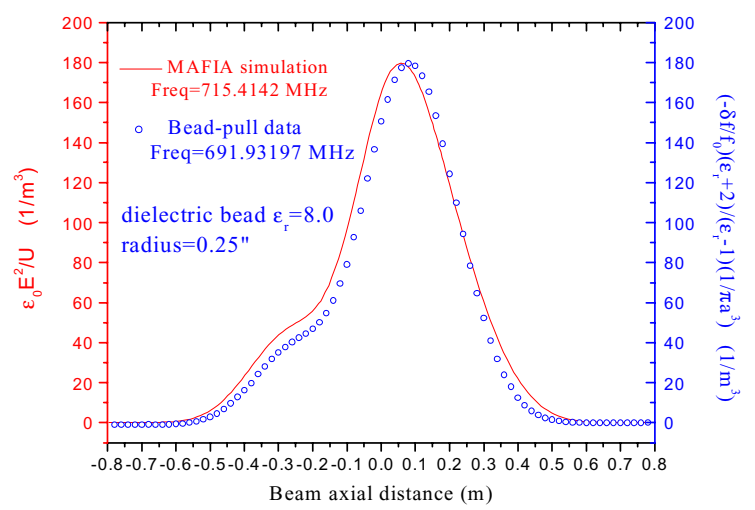

Figure 8: On-axis bead-pull measurement vs MAFIA simulation for the TM010 mode of polarimeter.

\section{CONCLUSION}

This analysis has demonstrated that MAFIA simulation can be used as a tool to check, understand and minimize shunt impedance of beam components. Both RHIC CNI polarimeter and Roman Pots have been designed under impedance limits of both injection and storage cavities. The heat load from the beam parasitic loss is acceptable.

\section{REFERENCES}

[1] T. Weiland et al., http://www.cst.de/.

[2] M. Zisman, et al., LBL-21270 UC-28.

[3] J. Wei, et al., RHIC/AP/145, BNL, Dec. 1997.

[4] J. Rose, RHIC/RF/27, BNL, Feb. 1995.

[5] H. Wang, H. Huang, RHIC/AP/178, Oct. 1999.

[6] H. Huang, et al., these proceedings

[7] S. Kurennoy, Phys. Rev. Special Topics: Accelerator and Beams, Vol. 2. 032001, 1999.

[8] S. Peggs, W. MacKay, "Collective Instabilities in RHIC", Sept. 1994.

[9] V. Mane and S. Peggs, RHIC/AP/34, BNL, Oct. 1994. 